\title{
Validação da versão em português do Mini-Inventário de Fobia Social (M ini-SPIN)
}

\author{
Validation of the Portuguese version \\ of the M ini-Social Phobia Inventory (M ini-SPIN)
}

Gustavo J oséFonseca D'El Rey ${ }^{1}$

CláudiaWilmor $\mathrm{M}$ atos ${ }^{1}$

${ }^{1}$ Programa de Fobia Social, Centro de Pesquisas e

Tratamento deTranstornos deAnsiedade. RuaBom Jesus 274-B, Água Rasa. 03344-000 São Paulo SP. g.delrey@bol.com.br
Abstract Social phobia (also known as social anxiety disorder) is a severe mental disorder that brings distress and disability. The aim of this study was validate to the Portuguese language the M ini-Social Phobia Inventory (M ini-SPIN) in a populational sample. We performed a discriminative validity study of the M ini-SPIN in a sample of 644 subjects ( $M$ iniSPIN positive group: $\mathrm{n}=218$ and control/negative group: $n=426$ ) of a study of anxiety disorders' prevalencein the city of Santo AndréSP. ThePortuguese version of the Mini-SPIN (with score of 6 points, suggested in the original English version) demonstrated a sensitivity of $95.0 \%$, specificity of $80.3 \%$, positive predictive value of $52.8 \%$, negative predictive value of $98.6 \%$ and incorrect classification rate of $16.9 \%$. With score of 7 points, was observed an increase in the specificity and positive predictivevalue $(88.6 \%$ and $62.7 \%)$, while the sensitivity and negative predictive value ( $84.8 \%$ and $96.2 \%$ ) remained high. The Portuguese version of the M ini-SPIN showed satisfactory psychometric qualities in terms of discriminative validity. In this study, the cut-off of 7, was considered to be the most suitable to screening of the generalized social phobia.

Key words Social phobia, Psychometrics, Validity, Rating scales, M ini-SPIN
Resumo A fobia social (também conhecida como transtorno de ansi edade social) é um grave transtorno mental que traz sofrimento e incapacitação. 0 objetivo deste estudo foi validar para a língua portuguesa o M ini-Inventário deFobia Social ( $M$ iniSPIN ) em uma amostra da população. Foi realizado um estudo da validade discriminativa do M iniSPIN em uma amostra de644 pessoas ( grupo positivo para o M ini-SPIN : $n=218$ e grupo controle/ negativo: $n=426$ ) de um estudo de prevalência de transtornos de ansiedade na cidade de Santo André (SP). A versão em português do M ini-SPIN (com escore de 6 pontos, sugerido na versão original em inglês) demonstrou uma sensibilidade de $95,0 \%$, especificidade de $80,3 \%$, valor preditivo positivo de $52,8 \%$, valor preditivo negativo de $98,6 \%$ e taxa de classificação incorreta de 16,9\%. Com escores de 7 pontos, foi observado um aumento na especificidade e no valor preditivo positivo ( $88,6 \%$ e $62,7 \%$ ), sendo que a sensi bilidade e 0 valor preditivo negativo $(84,8 \%$ e $96,2 \%)$ mantiveram-se altos. A versão em português do M ini-SPIN apresentou qualidades psicométricas satisfatórias em termos de validade discriminativa. N este estudo, o ponto de corte igual a 7 mostrou-se mais adequado para a identificação da fobia social generalizada.

Palavras-chave Fobia social, Psicometria, Validade, Escalas de avaliação, M ini-SPIN 
Introdução

A fobia social (também conhecida como transtorno de ansiedade social) é um transtorno mental severo que traz sofrimento e perdas de oportunidades a seus portadores, além de apresentar uma alta comorbidade com outros transtornos mentais, principalmente 0 subtipo generalizado $0^{1,2}$.

Felizmente, a fobia social responde favoravelmente a uma série de intervenções farmacológicas e psicológicas, como a terapia cognitivo-comportamental ${ }^{3-5}$.

Apesar do sofrimento e das boas opções de tratamento, a fobia social ainda é pouco reconhe cida pelos profissionais de saúde, inclusive os de saúde mental 6 . D evido a este fato, muitos pacientes não recebem 0 tratamento adequado $0^{7,8}$.

As escal as de avaliação para fobia social vieram a suprir em parte essa lacuna relacionada ao fraco reconhecimento do transtorno?.

Entre as escalas de avaliação para a identificação da fobia social generalizada, está o M ini-Inventário de Fobia Social (M ini-SPIN). Esteinstrumento foi desenvolvido pela Dra. Connor e seu grupo ${ }^{10}$, sendo derivado do Inventário de Fobia Social $(\text { SPIN })^{11}$.

O M ini-SPIN contém apenas três (itens 6, 9 e 15) dos dezessete itens do SPIN, que em estudo empírico mostraram-se indicativos da fobia social generalizada. $\mathrm{O} \mathrm{M}$ ini-SPIN éum instrumento autoaplicável composto por três itens que avaliam medo de constrangimento e evitação. A pontuação total varia de 0 a 12 . $N$ a versão em inglês, escores de seis pontos ou mais são indicativos da presença da fobia social generalizada. No estudo original, o M iniSPIN (ponto de corte igual a seis) apresentou sensibilidade de $88,7 \%$, especificidade de $90,0 \%$, valor preditivo positivo de $52,5 \%$ e valor preditivo negativo de $98,5 \%^{10}$. Atéo presentemomento eatéonde sabemos, existem dois estudos da versão em português do M ini-SPIN . U m estudo relativo à avaliação da consistência interna (que foi considerada boa) em uma amostra de universitários ${ }^{12}$ e outro relativo à validade discriminativa do instrumento em uma amostra de estudantes de terceiro grau ${ }^{13}$.

Este estudo teve como objetivo avaliar a validade discriminativa da versão em língua portuguesa do M ini-Inventário de Fobia Social (M iniSPIN) em uma amostra populacional da cidade de Santo André(SP).
Métodos

Versão em português

Utilizamos a mesma versão em português do Mini-SPIN descrita em estudo anterior sobre a consistência interna do instrumento (para maiores detalhes, ver D'El Rey et al. ${ }^{12}$ ).

\section{Participantes}

A população desta pesquisa foi constituída de 4.517 indi víduos adultos de ambos os sexos da cidade de Santo André (SP), que estavam participando de um estudo sobre a prevalência ao longo da vida e em doze meses de transtornos de ansiedade na população geral (tese de doutorado da segunda autora deste trabal ho, ainda em processo definalização).

\section{Procedimentos}

Juntamente com o material do estudo de prevalência de transtornos de ansiedade, foi entregue aos participantes ( $n=4.517)$ a versão em língua portuguesa do M ini-SPIN e o Inventário de Depressão Beck (BDI) ${ }^{14}$. Em relação ao ponto decorte do BDI, recomenda-se escores acima de 15 para detectar sintomas disfóricos em amostras não-clínicas ${ }^{15}$. Os indivíduos que apresentaram escores de seis ou mais pontos (ponto de corte sugerido na versão original em inglês) no M ini-SPIN e concordaram em continuar participando da presente pesquisa ( $n=218$ ) foram entrevistados por telefone para a confirmação do diagnóstico de fobia social generalizada através do módulo para fobia social contido na Entrevista Clínica Estruturada para o DSM - IV (SCID-I/P 2.0) ${ }^{16}$. Todas as entrevistas foram conduzidas por profissionais de saúde mental experientesecom treinamento na aplicação da SCID e de escal as de avaliação. Estes entrevistadores não tinham conhecimento dos escores do M ini-SPIN das pessoas que estavam entrevistando. Para comparação, uma amostra ran domizada de pessoas que apresentaram escores inferiores a seis pontos no Mini-SPIN e escores acima de quinze pontos no BDI ( $n=251$ ) eindivíduos queapresentaram escores inferiores a seis pontos no M ini-SPIN e escores inferiores a quinze pontos no BDI ( $n=175$ ) foram selecionados para comporem dois grupos controles, sendo entrevistados da mesma maneira. A amostra total compreendeu 644 pessoas, sendo 218 no grupo positivo para o M ini-SPIN (escores de seis pontos ou mais) e 426 pessoas nos grupos controles/negativo para o M ini-SPIN. 


\section{Análise dos dados}

A validação da versão em português do M iniSPIN foi realizada através da validade discriminativa (sensibilidade, especificidade, valores preditivos positivo e negativo e taxa de classificação incorreta), ou seja, neste tipo de validade, procurase avaliar o grau com que o instrumento discrimina entre pessoas que diferem em determinadas características de acordo com um critério padrão - padrão ouro ${ }^{17}$.

\section{Questões éticas}

Esta pesquisa esteve de acordo com a Resolução n 196/96 do Consel ho Nacional deSaúde para estudos envolvendo seres humanos. A presente pesquisa foi aprovada pelo Comitê de Ética em Pesquisa do Centro de Pesquisas e Tratamento de Transtornos de Ansiedade de São Paulo (SP).

\section{Resultados}

Características da amostra e escores do Mini-SPIN (grupo positivo e controles)

Seiscentas e quarenta e quatro pessoas participaram desta etapa do estudo de validação em língua portuguesa do M ini-SPIN, sendo 218 no grupo positivo para o Mini-SPIN (M S-P) e 426 nos grupos controles $(\mathrm{GC})$. As idades variaram entre 18 e 63 anos, sendo a média de 40,1 anos (DP = 13,2 ) no grupo MS-P e entre 18 e 59 anos, com média de 43,7 anos ( $D P=12,3$ ) nos GC. Os escores do M ini-SPIN na amostra M S-P variaram entre 6 a 11 pontos, com a média de 8,2 pontos ( $D P=1,7$ ) e os escores para os GC variaram de 0 a 5 pontos, com a média de 3,3 pontos ( $D P=1,2$ ). As características sociodemográficas dos sujeitos estão sumarizadas na Tabela 1 para uma melhor visualização.

Validade discriminativa do Mini-SPIN

Através das entrevistas realizadas com a SCID, o diagnóstico de fobia social foi confirmado em $115(52,8 \%)$ das 218 pessoas que estavam no grupo positivo para o Mini-SPIN (M S-P), ao passo

Tabela 1. Características sócio-demográficas dos participantes $(n=644)$

\begin{tabular}{|c|c|c|c|}
\hline \multirow{2}{*}{ Características } & MS-P & GC & Total \\
\hline & $\mathrm{N}(\%)$ & $\mathrm{N}(\%)$ & $\mathrm{N}(\%)$ \\
\hline \multicolumn{4}{|l|}{ Gênero } \\
\hline Masculino & $97(44,5)$ & $184(43,2)$ & $281(43,6)$ \\
\hline Feminino & $121(55,5)$ & $242(56,8)$ & $363(56,4)$ \\
\hline \multicolumn{4}{|l|}{ Raça } \\
\hline Branca & $182(83,5)$ & $373(87,6)$ & $555(86,2)$ \\
\hline Negra & $25(11,5)$ & $21(4,9)$ & $46(7,1)$ \\
\hline Amarela & $11(5,0)$ & $32(7,4)$ & $43(6,7)$ \\
\hline \multicolumn{4}{|l|}{ Estado civil } \\
\hline Solteiro & $87(39,9)$ & $131(30,8)$ & $218(33,8)$ \\
\hline Casado & $119(54,6)$ & $266(62,4)$ & $385(59,8)$ \\
\hline Outro & $12(5,5)$ & $29(6,8)$ & $41(6,4)$ \\
\hline \multicolumn{4}{|l|}{ Escolaridade } \\
\hline 10 grau incompleto & $13(6,0)$ & $35(8,2)$ & $48(7,5)$ \\
\hline 1o grau completo & $51(23,4)$ & $64(15,0)$ & $115(17,9)$ \\
\hline 2으 grau incompleto & $70(32,1)$ & $97(22,8)$ & $167(25,9)$ \\
\hline $2 \varrho$ grau completo & $62(28,4)$ & $159(37,3)$ & $221(34,3)$ \\
\hline Superior incompleto & $14(6,4)$ & $42(9,9)$ & $56(8,7)$ \\
\hline Superior completo & $8(3,7)$ & $29(6,8)$ & $37(5,7)$ \\
\hline \multicolumn{4}{|l|}{ O cupação } \\
\hline Atividade remunerada & $127(58,3)$ & $321(75,4)$ & $448(69,5)$ \\
\hline Atividade não-remunerada & $82(37,6)$ & $84(19,7)$ & $166(25,8)$ \\
\hline Estudante & $9(4,1)$ & $21(4,9)$ & $30(4,7)$ \\
\hline
\end{tabular}

Nota: M S-P $=$ M ini-SPIN positivo $(n=218) ; G C=$ grupos controles $(n=426)$ 
queo diagnóstico foi refutado em $103(47,2 \%)$ pessoas dentro desse grupo positivo. A não presença do diagnóstico de fobia social foi confirmada em $420(98,6 \%)$ das 426 pessoas que estavam dentro dos grupos controles (negativo) para o M ini-SPIN $(G C)$, enquanto que o diagnóstico de fobia social estava presente em apenas $6(1,3 \%)$ pessoas desse grupo negativo para 0 instrumento.

A validade discriminativa da versão em língua portuguesa do M ini-SPIN, utilizando-se o ponto de corte de 6 sugerido na versão original em inglês, demonstrou que o instrumento apresenta sensibilidadede $95,0 \%$, especificidadede $80,3 \%$, valor pre ditivo positivo de $52,8 \%$, valor preditivo negativo de $98,6 \%$ e uma taxa de classificação incorreta de $16,9 \%$. A sensibilidade e a especificidade foram similares tanto para os homens $(94,7 \%$ e $80,1 \%$, respectivamente) como para as mulheres $(95,2 \%$ e $80,4 \%$, respectivamente).

Com escores de 7 pontos, foi observado um aumento na especificidade e no valor preditivo positivo ( $88,6 \%$ e $62,7 \%$ ), sendo quea sensibilidade e 0 valor preditivo negativo $(84,8 \%$ e $96,2 \%)$ mantiveram-se altos. A Tabela 2 apresenta a sensibilidade, especificidade, valores preditivos positivo e negativo e taxa de classificação incorreta para a versão em português do M ini-SPIN com diversos pontos de corte, para uma melhor comparação.

\section{Discussão}

Infelizmente, a fobia social ainda épouco reconhecida pelos profissionais de saúde, apesar das boas opções de tratamentos psicoterápicos e farmacológicos existentes ${ }^{3-5}$.

A versão em português do Mini-SPIN apresentou boas qualidades psicométricas relacionadas à validade discriminativa. Com o ponto de corte igual a seis, sugerido na versão original em inglês, 0 instrumento apresentou sensibilidade, especificidade, valores preditivo positivo e negativo semelhantes à versão original do M ini-SPIN, embora com escore de sete pontos tenha ocorrido um aumento na especificidade e valor preditivo positivo do inventário, mantendo-se a sensibilidade e o valor preditivo negativo altos e com diminuição da taxa de classificação incorreta (Tabela 2), aumentando a validade discriminativa, semeIhante aos achados do estudo de Osório et al. ${ }^{13}$.

I gualmente ao estudo original da versão em inglês, a versão em língua portuguesa do M iniSPIN apresentou um valor preditivo positivo relativamentebaixo utilizando o ponto de corteigual a 6, embora o valor preditivo negativo a sensibilidade e especificidade tenham sido altas. Connor et al. ${ }^{10}$ sugerem que, a partir de escores de seis pontos, o clínico deva investigar com outras questões a presença da fobia social generalizada. Concordamos com Osório et al..$^{13}$ e Wilson ${ }^{18}$, quanto à realização de outros estudos para uma melhor determinação do ponto de corte do Mini-SPIN, embora os resultados deste nosso estudo sugiram que a versão em português do M ini-SPIN apresente uma discriminância considerada boa com 0 ponto de corte igual a sete (bons valores para a sensibilidade, especificidade, VPP eVPN).

Por ser de fácil preenchimento pelo paciente e rápida correção, o M ini-SPIN pode ser utilizado em serviços de atenção primária em saúde e ambulatórios específicos de saúde mental, para que os pacientes com fobia social possam ser identificados e encaminhados para o tratamento adequado. Ele também pode ser incorporado em protocolos que avaliam aspectos de saúde mental em múltiplas condições médicas gerais e em estudos deprevalência ${ }^{10}$.

Os resultados encontrados neste estudo sugerem que a versão em português do M ini-SPIN avaliados em uma amostra populacional de um estudo

Tabela 2. Validade discriminativa do M ini-SPIN com vários pontos de corte.

\begin{tabular}{lccccc} 
Ponto decorte & Sensibilidade & Especificidade & $\begin{array}{c}\text { Valor preditivo } \\
\text { positivo }\end{array}$ & $\begin{array}{c}\text { Valor preditivo } \\
\text { negativo }\end{array}$ & $\begin{array}{c}\text { Taxa de classificação } \\
\text { incorreta }\end{array}$ \\
\hline 6 & 95,0 & 80,3 & 52,8 & 98,6 & 16,9 \\
7 & 84,8 & 88,6 & 62,7 & 96,2 & 12,1 \\
8 & 76,4 & 92,6 & 72,4 & 93,9 & 10,7 \\
9 & 70,3 & 95,6 & 82,2 & 91,8 & 10,1 \\
10 & 68,5 & 97,9 & 92,6 & 89,2 & 10,1 \\
\hline
\end{tabular}


de prevalência de transtornos de ansiedade apresentou qualidades psicométricas satisfatórias, visto que instrumentos traduzidos e adaptados para outras culturas podem ter sua estrutura modificada ${ }^{19}$.

Finalizando, este trabal ho não esgota as possibilidades das análises das propriedades psicomé tricas da versão em língua portuguesa do M iniSPIN, assim como estudos com outras populações (crianças, adolescentes, etc.) são necessários, parauma maior confirmação da val idade do M iniSPIN em língua portuguesa.

\section{Colaboradores}

GJF D'El Rey participou de toda a preparação da introdução, métodos, resultados e discussão. CW $M$ atos participou da preparação do método, re sultados e discussão.

\section{Consideraçõesfinais}

A versão em português do M ini-SPIN apresentou qualidades psicométricas satisfatórias em termos de validade discriminativa. N este estudo, o ponto de corte igual a sete mostrou-se mais adequado para a identificação da fobia social generalizada na população estudada. Pesquisas que verifiquem outras qualidades psicométricas do M ini-SPIN e com outras populações são necessárias, sendo este um campo promissor para futuros estudos aqui no Brasil.

\section{Referências}

1. Kessler RC, Stein MB, Berglund P. Social phobia subtypes in the National Comorbidity Survey. Am J Psychiatry 1998; 155(5):613-619.

2. D'El Rey GJF, Pacini CA. Comorbidade com a dependência de substâncias nos subtipos da fobia social. Arq Ciênc Saúde U nipar 2005; 9(3):207-210.

3. Gould RA, Buckminster A, Pollack PM, Yap RT. Cognitive-behavioral and pharmacological treatment for social phobia: a meta-analysis. Clin Psycho Sci Pract 1997; 4(3):296-306.

4. Heimberg RG. Current status of psychotherapeutic interventions for social phobia. J Clin Psychiatry 2001; 62(Suppl 1):36-42.

5. D'El Rey GJF. Terapia de exposição: um tratamento eficaz para fobia social. Psychiatry On Line Brazil. 2007 [acessado 2007 mar 11]; 12(2):[cerca de 5 p.]. Disponível em: http://www.polbr.med.br/ano07/art0207.php

6. Lamberg L. Social phobia: not just another name for shyness. JAM A 1998; 280(8):11-16.

7. Weiler E, Bisserbe JC, Boyer P, Leonine JP, Lecrubier $Y$. Social phobia in general health care: an unrecognised, untreated, disabling disorder. Br J Psychiatry 1996; 168(2):169-174.

8. Katzelnick DJ, Kobak KA, DeLeire T, Henk HJ, Greist JH, Davidson JRT, Schneier FR, Stein M B, Helstad CP. Impact of generalized social anxiety disorder in managed care. Am J Psychiatry 2001; 158(12):1999-2007.

9. D'El Rey GJF, Almeida IP. Transtornos fóbicos na atualidade. Integ Ens Pesq Ext 2002; 8(28):25-29.

10. Connor KM, Kobak KA, Churchill LE, Katzelnick D, Davidson JRT. M ini-SPIN : a brief screening assessment for generalized social anxiety disorder. Depress Anxiety 2001; 14(2):137-140. 
11. Connor KM, Davidson JRT, Churchill LE, Sherwood A, Foa EB, Weisler RH. Psychometric properties of the Social Phobia Inventory (SPIN): a new self-rating scale. Br J Psychiatry 2000; 176(4):379-386.

12. D'El Rey GJF, Lacava JPL, Cardoso R. Consistência interna da versão em português do M ini-Inventário de Fobia Social (M ini-SPIN). Rev Psiquiatr Clín 2007; 34(6):266-269.

13. Osório FL, Crippa JA, Loureiro SR. A study of the discriminative validity of a screening toll (M ini-SPIN) for social anxiety disorder applied to Brazilian university students. Eur Psychiatry 2007; 22(4):239-243.

14. Cunha JA. Manual em português das escalas Beck. São Paulo: Casa do Psicólogo; 2001.

15. Kendall PC, Hollon SD, Beck AT, Hammen $\mathrm{Cl}$, Ingram RE. Issues and recommendations regarding use of the Beck Depression Inventory. Cogn Ther Res 1987; 11(3):289-299.

16. Tavares M. Entrevista clínica estruturada para o DSM IV: transtornos do eixo 1 - edição para pacientes (SCIDI/P 2.0). Brasília: Instituto de Psicologia, Universidade de Brasília; 1996.

17. M enezes PR. Validade e confiabilidade das escalas de avaliação em psiquiatria. Rev Psiquiatr Clín 1998; 25(5):214-216.

18. Wilson I. Screening for social anxiety disorder in first year university students: a pilot study. Aust Family Phys 2005; 34(11):983-984.

19. Westermeyer J, Janca A. Language, culture, and psychopathology: conceptual and methodological issues. Trans Psychiatry 1997; 34(2):291-311.

Artigo apresentado em 12/07/2007

Aprovado em 03/04/2008 